\title{
Atributos físicos e estoques de carbono do solo sob diferentes usos da terra em Rondônia, Amazônia Sul-Ocidental
}

Adriano Reis Prazeres Mascarenhas ${ }^{1 *}$, Marta Silvana Volpato Sccoti ${ }^{1}$, Rafael Rodolfo Melo ${ }^{2}$, Fernando Luíz de Oliveira Corrêa ${ }^{3}$, Emanuel Fernando Maia de Souza ${ }^{1}$, Reginaldo Almeida Andrade ${ }^{1}$, Anderson Cristian Bergamin $^{1}$, Manfred Willy Müller $^{4}$

${ }^{1}$ Universidade Federal de Rondônia, Av. Norte Sul, CEP 76940-000, Rolim de Moura, RO, Brasil

${ }^{2}$ Universidade Federal de Mato Grosso, Av. Alexandre Ferronato, CEP 78550-000, Sinop, MT, Brasil

${ }^{3}$ Comissão Executiva do Plano da Lavoura Cacaueira, Superintendência de Desenvolvimento da Região Cacaueira do Estado de Rondônia, BR 364, Km 2,5, CEP 76920-000, Ouro Preto do Oeste, RO, Brasil

${ }^{4}$ Comissão Executiva do Plano da Lavoura Cacaueira, Diretoria Geral, Rua "G", Setor Sudoeste, Campus do INMET, CEP 70680-900, Brasília, DF, Brasil

"Autor correspondente:

adriano.mascarenhas@unir.br

Termos para indexação:

Agroecossistema

Física do solo

Sustentabilidade

Index terms:

Agroecosystems

Soil physics

Sustainability

Histórico do artigo:

Recebido em 08/06/2016

Aprovado em 22/03/2017

Publicado em 31/03/2017

doi: $10.4336 / 2017 . p f b .37 .89 .1295$
Resumo - Objetivou-se estudar o comportamento dos atributos físicos e dos teores e estoques de carbono do solo em sistema agroflorestal multiestratificado (SAF), comparativamente a diferentes modalidades de uso solo, sendo elas o cultivo de cacaueiro sob manejo agroecológico (CAC), pastagem cultivada (PAS) e floresta nativa (FLN). A área de estudo localiza-se na estação experimental da CEPLAC em Ouro do Preto do Oeste, RO. Nos quatro tratamentos foram instaladas, ao acaso, cinco parcelas de $20 \mathrm{~m}$ x $30 \mathrm{~m}$. Nessas parcelas, foram coletadas amostras de solo indeformadas e deformadas nas profundidades de 0 a $20 \mathrm{~cm}$ e 20 a $40 \mathrm{~cm}$, para determinação dos atributos fiscos e estoque de carbono no solo. Os valores de densidade do solo no SAF, CAC e PAS apresentaram-se elevados e influenciaram negativamente o espaço poroso do solo. Os estoques de $\mathrm{C}$ foram influenciados pelos diferentes agroecossistemas, sendo que na camada de 0 a $20 \mathrm{~cm}$ foram de $\left(\mathrm{Mg} \mathrm{m}^{-3}\right)$ : 24,79 (SAF); 42,71 (CAC); 41,30 (PAS); 36,07 (FLN), e na camada de 20 a $40 \mathrm{~cm}$ foram (Mg m$\left.{ }^{-3}\right): 17,26$ (SAF); 31,06 (CAC); 22,31 (PAS); 25,49 (FLN). Todos os sistemas avaliados apresentaram resultados esperados para estoque de $\mathrm{C}$ no solo, no entanto, arranjos espaciais que favoreçam o aporte de matéria orgânica poderiam contribuir para a elevação dos estoques de carbono do solo em sistemas agroflorestais.

\section{Physical attributes and soil carbon stocks under different land use in Rondonia State, South Western Amazonia}

\begin{abstract}
The objective of this study was to investigate the behavior of physical attributes and soil carbon stocks in multi-stratified agro forestry system (AFS) compared to different types of land use, including cocoa plantation in agroecological management (CAC), pasture (PAS) and native forest (FLN). The treatments were installed at CEPLAC experimental station in Ouro Preto do Oeste, Rondonia State. Five plots of $20 \mathrm{~m} \times 30 \mathrm{~cm}$ were installed at random in each of the four different land use. On those plots, disturbed and undisturbed soil samples in layers of $0-20 \mathrm{~cm}$ and $20-40 \mathrm{~cm}$ depth were collected for determination of physical attributes and soil carbon stock. Soil density in AFS, CAC and PAS were high and promoted negative influences on soil porosity. Soil carbon stocks were influenced by different land use modality,

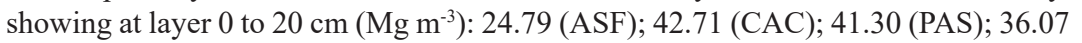

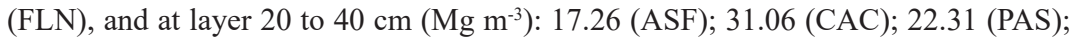
25.49 (FLN). All evaluated systems presented expected soil carbon stocks, however spatial arrangements that favor the input of organic matter may contribute to increase soil carbon stocks in agroforestry systems.
\end{abstract}




\section{Introdução}

Práticas de uso e conservação do solo podem conter ações que minimizem uso de combustíveis fósseis, desmatamento e queima de biomassa vegetal. Dessa forma, agroecossistemas de elevada eficiência ambiental, social e econômica são plausíveis. Parron et al. (2015) reforçam essa ideia ao afirmarem que sistemas de produção conservacionistas, quando comparados às práticas intensivas da agricultura convencional, têm grande potencial na redução das emissões de gases de efeito estufa (GEEs), diminuição do consumo de combustíveis durante o processo de produção e ainda podem elevar os estoques de carbono orgânico do solo (COS), mantendo os serviços ambientais e atenuando os efeitos negativos provocados pelas mudanças climáticas.

Os sistemas agroflorestais se inserem nesse contexto, porque se considerada como excelente alternativa para mitigação de GEEs (Nair et al., 2009). Esses são amplamente utilizados no mundo, com o cultivo simultâneo de árvores, espécies agrícolas e/ou animais, de acordo com um arranjo espacial e/ou temporal e ainda produzem grande quantidade de biomassa, favorecendo o acúmulo de carbono (Altieri, 2012).

Além desses benefícios, pode-se atribuir aos SAFs o potencial de proteção dos solos, principalmente na recuperação de áreas degradadas. Segundo Pezarico et al. (2012), esse modo de produção tem apresentado contribuição significativa na melhoria dos atributos físico-químicos do solo. Entretanto, existem gargalos no entorno da temática agroflorestal, principalmente no que diz respeito a informações mais detalhadas sobre as características dos SAFs para promover melhor aproveitamento de seu potencial, principalmente na região amazônica, pois as pesquisas nesse sentido são incipientes (Magalhães et al., 2014).

Deste modo, faz-se necessário a investigação da eficiência ambiental dos SAFs em comparação a outros agroecossistemas na região amazônica, para subsidiar práticas de manejo que causem menor impacto ao ambiente e aumentem a resiliência das paisagens. Costa Junior (2008) e Silva et al. (2015) sugerem que para se obter informações dessa magnitude torna-se necessário o uso de indicadores de qualidade ambiental, dentre os quais, destacam-se os parâmetros físicos da qualidade do solo, que são fundamentais à compreensão dos processos de degradação do solo e os estoques de COS, por estarem diretamente relacionados aos atributos químicos, físicos e biológicos do solo.
Diante desse contexto, objetivou-se neste trabalho avaliar a variação dos atributos físicos (densidade e espaço poroso do solo) e dos estoques de carbono do solo em sistema agroflorestal multiestratificado, comparativamente a diferentes modalidades de uso solo e um fragmento remanescente de floresta primária.

\section{Material e métodos}

A pesquisa foi realizada na "Estação Experimental Ouro Preto", pertencente à Comissão Executiva do Plano da Lavoura Cacaueira do Ministério da Agricultura, Pecuária e Abastecimento, localizada no Município de Ouro Preto do Oeste, RO (1043'6,36”S e 62'13'22,17' 'W), km 2,5 da Rodovia Federal BR 364.

O solo da região é classificado como Argissolo Vermelho-Amarelo Eutrófico (PVAe) (Santos et al., 2013). O clima enquadra-se no tipo Am, conforme classificação climática de Köppen-Geiger (Alvares et al., 2013), com temperatura média anual de $26{ }^{\circ} \mathrm{C}$ e precipitação pluviométrica média anual de $2.300 \mathrm{~mm}$. A umidade relativa do ar varia entre 80 e $90 \%$ de novembro a maio e em julho e agosto permanece em torno de $75 \%$ (Brasil, 2007). A fitogeografia do local possui fragmentos de Floresta Ombrófila Densa Submontana com Dossel Uniforme (Dsu), Floresta Ombrófila Densa Submontana com Dossel Emergente (Dse) e Floresta Ombrófila Aberta Submontana com Palmeiras (Asp) (IBGE, 2012).

Foram selecionadas na estação experimental quatro áreas, com as seguintes características: sistema agroflorestal multiestratificado (SAF), cultivo de cacaueiro como manejo agroecológico (CAC), pastagem (PAS) e floresta nativa (FLN).

O SAF foi instalado em uma área de $80 \mathrm{~m}$ de largura por $125 \mathrm{~m}$ de comprimento há 20 anos. A cobertura anterior desse sistema era constituída por vegetação em processo de regeneração natural, que após supressão deu lugar aos plantios de milho, algodão e feijão. A área recebeu calagem e adubação nos primeiros anos de cultivo. Posteriormente, implantaram-se no sistema espécies agrícolas frutíferas: araçazeiro-boi (Eugenia stipitata Mc Vaugh.), cacaueiro (Theobroma cacao L.), cajá-mirim (Spondia mombim L.), coqueiro (Cocos nucifera L.), fruteira-pão (Arthocarpus altilis P.), laranjeira (Citrus sinensis L.), mangueira (Mangifera indica L.) e pupunheira (Bactris gasipaes Kunth) e florestais: canela (Cinnamomum zeylanicum J.Presl), cumaru-ferro (Dipteryx odorata Aublet. Willd.), freijó (Cordia goeldiana Huber.), mogno africano 
(Khaya senegalensis), pinho-cuiabano (Schizolobium amazonicum Huber x Ducke) e teca (Tectona grandis L.f.). Todas as espécies estão plantadas em renques duplos com espaçamento de $5 \mathrm{~m}$ x 2,5 m (Figura 1).
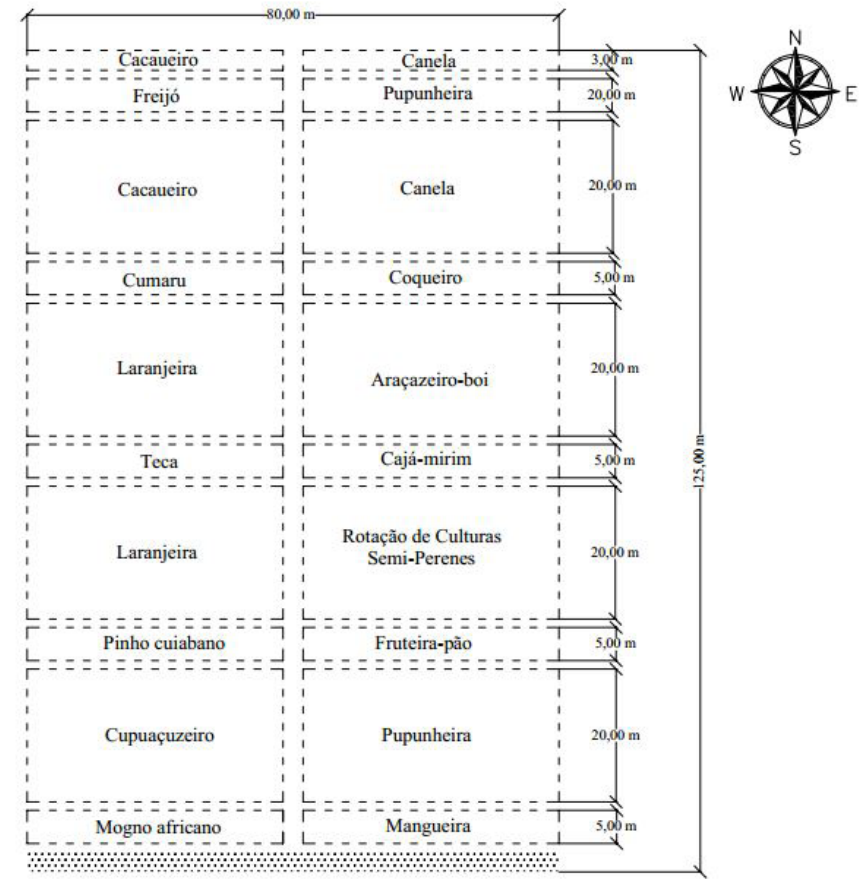

Figura 1. Representação do arranjo espacial das espécies que constituem o sistema agroflorestal multiestratificado na Estação Experimental da CEPLAC em Ouro Preto do Oeste, RO.

O local correspondente ao CAC é resultante da conversão de uso do solo realizada a partir de uma lavoura convencional de cacaueiros, com 20 anos, na qual realizou-se calagem e adubações. Esse histórico de uso explica os teores de cálcio e fósforo revelados na Tabela 1. O atual sistema de uso do solo caracteriza-se por apresentar cacaueiros ( $T$. cacao $)$ no espaçamento $3 \mathrm{~m} \times 3 \mathrm{~m}$ com manejo agroecológico, ou seja, nos tratamentos fitossanitários não são utilizados agroquímicos industriais e a adubação é estritamente orgânica, realizada por meio de compostagem. A idade desse sistema é de 2 anos. Os cacaueiros são sombreados por bananeiras (Musa sp.) no espaçamento $3 \mathrm{~m} \times 3$ $\mathrm{m}$ e pela leguminosa arbustiva gliricídia (Gliricidia sepium Jacq. Steud) com espaçamento de $6 \mathrm{~m}$ x $6 \mathrm{~m}$, a qual recebe podas periódicas, de modo que o material resultante fique depositado na superfície do solo.

A PAS utilizada tem aproximadamente 15 anos e é formada, predominantemente, pela gramínea forrageira Brachiaria sp., manejada de forma extensiva, ou seja, com baixo nível tecnológico.

A área considerada como referência (FLN), é um fragmento remanescente de Floresta Ombrófila Aberta Submontana com Palmeiras (Asp) (IBGE, 2012), que é adjacente a uma unidade de conservação municipal (Parque Chico Mendes).

De modo geral, nenhum dos agroecossistemas apresentou registro de mecanização ou tráfego de veículos. Na Tabela 1, são apresentadas as características físicas e químicas do solo das áreas consideradas para o estudo. Há uma tendência de redução de argila no perfil do solo, como pode ser observado para o ambiente do SAF e PAS, entretanto não configuram classes de solos diferentes dos ambientes CAC e FLN, pois o Argissolo Vermelho Amarelo Eutrófico apresenta saturação de bases acima de $50 \%$ nos primeiros $100 \mathrm{~cm}$ do horizonte BA (Santos et al., 2013).

Tabela 1. Características físicas e químicas do solo nas camadas de 0 a $20 \mathrm{~cm}$ e 20 a $40 \mathrm{~cm}$ em diferentes sistemas de uso do solo em Ouro Preto do Oeste, RO.

\begin{tabular}{|c|c|c|c|c|c|c|c|c|c|c|c|c|}
\hline \multirow{2}{*}{$\begin{array}{l}\text { Sistemas de } \\
\text { uso do solo }\end{array}$} & \multirow{2}{*}{$\begin{array}{c}\text { Camadas } \\
(\mathbf{c m})\end{array}$} & \multirow{2}{*}{$\begin{array}{c}\mathbf{p H} \\
\left(\mathrm{H}_{2} \mathrm{O}\right)\end{array}$} & \multirow{2}{*}{$\begin{array}{c}\mathbf{p H} \\
\left(\mathrm{CaCl}_{2}\right)\end{array}$} & $\mathbf{V}$ & $\mathbf{P}$ & $\mathbf{K}^{2+}$ & $\mathrm{Ca}^{2+}$ & $\mathrm{Mg}^{2+}$ & $\mathbf{A} \mathbf{l}^{3+}$ & Areia & Silte & \multirow[t]{2}{*}{ Argila } \\
\hline & & & & $\%$ & \multicolumn{2}{|c|}{$\mathrm{mg} \mathrm{dm}^{-3}$} & \multicolumn{3}{|c|}{$\mathrm{cmol}_{\mathrm{c}} \mathbf{d m}^{-3}$} & & $\mathrm{~g} \mathrm{~kg}^{-1}$ & \\
\hline \multirow{2}{*}{ SAF } & $0-20$ & 5,80 & 5,20 & 59,10 & 2,50 & 41,00 & 2,00 & 0,42 & 0,00 & 661,00 & 128,00 & 211,00 \\
\hline & $20-40$ & 5,90 & 5,10 & 63,20 & 1,20 & 50,00 & 1,26 & 0,33 & 0,00 & 600,00 & 143,00 & 257,00 \\
\hline \multirow{2}{*}{ CAC } & $0-20$ & 6,70 & 6,40 & 78,60 & 6,90 & 94,00 & 7,71 & 0,78 & 0,00 & 476,00 & 177,00 & 347,00 \\
\hline & $20-40$ & 6,50 & 5,70 & 41,00 & 2,10 & 70,00 & 3,44 & 0,52 & 0,00 & 415,00 & 162,00 & 423,00 \\
\hline \multirow{2}{*}{ PAS } & $0-20$ & 5,60 & 4,60 & 34,20 & 2,00 & 83,00 & 1,32 & 0,29 & 0,06 & 615,00 & 128,00 & 257,00 \\
\hline & $20-40$ & 5,80 & 4,90 & 70,30 & 0,90 & 52,00 & 1,24 & 0,23 & 0,00 & 492,00 & 176,00 & 332,00 \\
\hline \multirow{2}{*}{ FLN } & $0-20$ & 5,70 & 5,10 & 58,70 & 1,40 & 83,00 & 3,43 & 0,63 & 0,00 & 415,00 & 162,00 & 423,00 \\
\hline & $20-40$ & 5,70 & 5,00 & 53,10 & 1,00 & 68,00 & 2,74 & 0,48 & 0,00 & 353,00 & 190,00 & 457,00 \\
\hline
\end{tabular}

Em que: $\mathrm{SAF}=$ sistema agroflorestal multiestratificado $\mathrm{CAC}=$ cacau sob manejo agroecológico; $\mathrm{PAS}=$ pastagem; FLN $=$ floresta natural; $\mathrm{V}=$ saturação por bases. 


\section{Amostragem do solo}

As coletas de solo foram realizadas em julho (período de estiagem na região) nas áreas de estudo (SAF, CAC, PAS e FLN), adotando-se um padrão de amostragem baseado nas dimensões do sistema agroflorestal multiestratificado (80 m x $125 \mathrm{~m})$. Em cada sistema de uso do solo, foram estabelecidas, ao acaso, 5 unidades amostrais com dimensões de $20 \mathrm{~m}$ x $30 \mathrm{~m}$, empregando-se o delineamento experimental inteiramente casualisado, pois as áreas apresentam as mesmas condições edafoclimáticas (Costa Junior, 2008). Foram consideradas 4 fontes de variação (sistemas de uso do solo) e 2 profundidades. As variáveis estudadas foram densidade do solo (DS), macro e microporosidade, porosidade total, textura do solo, teor e estoque de carbono orgânico do solo (COS).

\section{Atributos físicos do solo}

Para as determinações da densidade do solo (Ds), macroporosidade (Map), microporosidade (Mip) e porosidade total $(\mathrm{Pt})$, escavou-se em cada unidade amostral, de cada sistema de uso do solo, uma trincheira de $40 \mathrm{~cm}$ de largura, $70 \mathrm{~cm}$ de comprimento e $40 \mathrm{~cm}$ de profundidade. Em seguida, coletaram-se amostras com estrutura preservada em cilindros metálicos, medindo $60,28 \mathrm{~mm}$ de diâmetro interno e 40,52 $\mathrm{mm}$ de altura, com bordas cortantes. As amostras foram coletadas entre $0 \mathrm{e}$ $20 \mathrm{~cm}$ e entre 20 e $40 \mathrm{~cm}$ de profundidade.

Após as coletas, as amostras foram encaminhadas ao Laboratório de Solos da Universidade Federal de Rondônia, em Rolim de Moura, RO, onde foram previamente preparadas e submetidas à saturação, com a técnica de elevação gradual de água, submergindo-as cerca de dois terços da altura do anel. Esse procedimento foi necessário para determinação da macroporosidade (Equação 1), microporosidade (Equação 2) e porosidade total (Equação 3), adotando-se o método da mesa de tensão (Donagema et al., 2011). Para a obtenção da densidade do solo (Equação 4) utilizou-se a metodologia em que as amostras indeformadas são acondicionadas em estufa a $105^{\circ} \mathrm{C}\left( \pm 5^{\circ} \mathrm{C}\right)$ até apresentar massa constante (Claessen, 1997). Os valores encontrados para densidade foram utilizados para o cálculo do estoque de carbono no solo.

$$
\operatorname{Mip}=\left(\frac{a-b}{c}\right)
$$

$$
\begin{aligned}
& \text { Map }=\text { VS }- \text { MIP } \\
& \mathrm{Pt}=\text { Mip }+ \text { Map } \\
& \mathrm{Ds}=\frac{\mathrm{m}}{\mathrm{v}}
\end{aligned}
$$

Em que: Mip $=$ microporosidade $\left(\mathrm{m}^{3} \mathrm{~m}^{-3}\right) ; \mathrm{a}=$ massa de amostra após ser submetida a uma tensão de $60 \mathrm{~cm}$ de coluna de água $(\mathrm{kg}) ; \mathrm{b}=$ massa da amostra seca a $105^{\circ} \mathrm{C}(\mathrm{kg})$; c $=$ volume do cilindro $\left(\mathrm{m}^{3}\right)$; Map $=$ macroporosidade $\left(\mathrm{m}^{3} \mathrm{~m}^{-3}\right) ; \mathrm{Vs}=$ volume de saturação $\left(\mathrm{m}^{3} \mathrm{~m}^{-3}\right) ; \mathrm{Pt}=$ porosidade total $\left(\mathrm{m}^{3} \mathrm{~m}^{-3}\right) ; \mathrm{Ds}=$ densidade do solo $\left(\mathrm{Mg} \mathrm{m}^{-3}\right) ; \mathrm{m}=$ massa da amostra seca a $105^{\circ} \mathrm{C}$ $(\mathrm{Mg}) ; \mathrm{v}=$ volume do cilindro $\left(\mathrm{m}^{3}\right)$.

\section{Determinação dos teores e estoques de carbono}

Para se determinar o teor de carbono do solo, fezse necessário o preparo da amostra deformada, a fim de se obter terra fina seca ao ar (TFSA), por meio do destorroamento e peneiramento em malha de $2 \mathrm{~mm}$ (Donagema et al., 2011). Em seguida, triturou-se cerca de $20 \mathrm{~g}$ de TFSA em almofariz (pilão de porcelana) e peneirou-se o material em malha de $0,25 \mathrm{~mm}$ até que se observasse aspecto de pó.

Com esse material preparado, realizou-se a determinação de $\mathrm{C}$ por meio do método de oxidação via dicromato de potássio, ajustado para condições amazônicas por Silva et al. (2014), que consiste na adição sequencial de, aproximadamente, $0,5 \mathrm{~g}$ do solo em pó, aferido em balança com precisão de 0,$001 ; 10$ $\mathrm{mL}$ de dicromato de potássio $(0,166 \mathrm{M}) ; 10 \mathrm{~mL}$ de ácido sulfúrico concentrado; $3 \mathrm{~mL}$ de ácido ortofosfórico concentrado; $47 \mathrm{~mL}$ de água destilada ou deionizada com mais 10 gotas do indicador difenilamina $\left(10 \mathrm{~g} \mathrm{~L}^{-1}\right)$ em erlenmeyer de $125 \mathrm{~mL}$.

Após o resfriamento, titularam-se as amostras com sulfato ferroso amoniacal $(0,5 \mathrm{M})$, por meio de bureta digital e agitador magnético, até transição da cor da mistura, de roxo para verde, anotando-se ao final o volume gasto da solução. Deste modo, prosseguiu-se para o cálculo do teor de carbono do solo, utilizando-se a Equação 5. Os cálculos dos estoques de $\mathrm{C}$ total do solo foram realizados a partir dos teores de $\mathrm{C}$ juntamente com a densidade do solo, para cada camada amostrada, utilizando-se a metodologia de Veldkamp (1994), por meio da Equação 6. 


$$
\begin{aligned}
& \mathrm{C}=\frac{(\mathrm{BR}-\mathrm{AM}) \times 3,9 \times\left(\frac{10}{\mathrm{FT}}\right)}{\mathrm{MA}} \\
& \text { Est COT }=\frac{(\mathrm{C} \times \mathrm{Ds} \times \mathrm{e})}{10}
\end{aligned}
$$

Em que: $\mathrm{C}=$ carbono total da amostra $\left(\mathrm{g} \mathrm{kg}^{-1}\right) ; \mathrm{BR}=$ volume gasto de sulfato ferroso amoniacal para titular prova em branco $(\mathrm{mL}) ; \mathrm{AM}=$ volume gasto de sulfato ferroso amoniacal para titular a amostra $(\mathrm{mL}) ; \mathrm{FT}=$ fator de correção para dicromato de potássio; MA = massa de solo utilizada para determinação de $\mathrm{C}$, neste caso 0,5 g; Est COS =estoque de carbono orgânico em determinada profundidade $\left(\mathrm{Mg} \mathrm{ha}^{-1}\right)$; $\mathrm{Ds}=$ densidade do solo da profundidade $\left(\mathrm{g} \mathrm{cm}^{-3}\right) ; \mathrm{e}=$ espessura da camada considerada $(\mathrm{cm})$.

\section{Análise dos resultados}

O conjunto de dados foi submetido ao teste de normalidade de Shapiro-Wilk, a 5\% de significância e, posteriormente, com o propósito de se conhecer diferenças significativas entre as fontes de variação, aplicou-se a análise de variância (ANOVA) aos resultados. As médias foram comparadas pelo teste de Sccott-Knott ao nível de 5\% de probabilidade. Os dados de atributos físicos do solo e teores de carbono orgânico do solo também foram submetidos à análise de correlação de Pearson a $1 \%$ e $5 \%$ de probabilidade.

\section{Resultados e discussão}

Foi observada variação significativa entre as médias nas profundidades avaliadas ( 0 a $20 \mathrm{~cm}$ e 20 a $40 \mathrm{~cm}$ ), exceto para macroporosidade. Os valores médios de densidade do solo (Ds) entre os ambientes estudados estão apresentados na Tabela 2. De modo geral, esses resultados estão acima do valor crítico para Ds. Silva et al. (2015) indicam que valores de Ds acima de $1,40 \mathrm{Mg} \mathrm{m}^{-3}$ podem acarretar restrições ao crescimento radicular e infiltração de água no solo.

As médias de Ds na camada de 0 a $20 \mathrm{~cm}$ são semelhantes nas áreas de SAF, CAC e PAS, diferindo apenas da referência (FLN), que foi em média, 12\% menor que os demais valores encontrados (Tabela 2). No entanto, na camada de $20 \mathrm{~cm}$ a $40 \mathrm{~cm}$, a média de Ds no sistema agroflorestal multiestratificado diferiu dos demais usos de solo, apresentando o maior valor para o atributo $\left(1,72 \mathrm{Mg} \mathrm{m}^{-3}\right)$. Sugere-se que esse resultado seja decorrente do pouco revolvimento do solo e ao reduzido aporte de matéria orgânica observado em campo. Segundo Silva et al. (2008), os sistemas conservacionistas, nos quais os SAFs se incluem, caracterizam-se por apresentarem essas condições, o que pode favorecer o aumento da Ds nas camadas superficiais em relação aos sistemas convencionais.

Tabela 2. Densidade do solo (Ds) em diferentes sistemas de uso do solo em duas profundidades em Ouro Preto do Oeste,

\begin{tabular}{|c|c|c|c|c|c|c|}
\hline \multirow{2}{*}{$\begin{array}{l}\text { Atributo } \\
\left(\mathrm{Mg} \mathrm{m}^{-3}\right)\end{array}$} & \multirow{2}{*}{$\begin{array}{l}\text { Profundidades } \\
\text { (cm) }\end{array}$} & \multicolumn{4}{|c|}{ Sistemas de uso do solo } & \multirow{2}{*}{$\begin{array}{l}\text { CV } \\
(\%)\end{array}$} \\
\hline & & SAF & CAC & PAS & FLN & \\
\hline \multirow{2}{*}{ Ds } & $0-20$ & $1,63 \mathrm{a}$ & $1,56 \mathrm{a}$ & $1,55 \mathrm{a}$ & $1,43 b$ & 5,81 \\
\hline & $20-40$ & $1,72 \mathrm{a}$ & $1,59 \mathrm{~b}$ & $1,53 b$ & $1,51 \mathrm{~b}$ & 5,70 \\
\hline
\end{tabular}
RO.

Em que: $\mathrm{SAF}=$ sistema agroflorestal multiestratificado; $\mathrm{CAC}=$ cacau sob manejo agroecológico; PAS = pastagem; FLN = floresta natural, $\mathrm{CV}$ = coeficiente de variação. Médias na linha, seguidas de mesma letra, não diferem pelo teste de Sccott-Knott a 5\% de probabilidade.

Ainda em relação à camada de $20 \mathrm{~cm}$ a $40 \mathrm{~cm}$, a Ds no ambiente FLN apresentou a menor média $\left(1,51 \mathrm{Mg} \mathrm{m}^{-3}\right)$, provavelmente em função da elevada densidade de plantas e do acúmulo de serrapilheira na superfície do solo (Pezarico et al., 2012). Em geral, a Ds na camada de 20 a $40 \mathrm{~cm}$ apresentou a tendência de elevação dos valores à medida que se aumenta a profundidade no perfil do solo, conforme descrito por Sato (2005). Segundo esse autor, esse comportamento é natural e típico, devido às pressões exercidas nas camadas superficiais que promovem compactação e redução da matéria orgânica nas camadas mais profundas, reduzindo a porosidade do solo.

No entanto, percebe-se que apesar do SAF apresentar uma estrutura multiestratificada, que simula a sucessão de florestas naturais, não atingiu valores de Ds próximos ao da FLN, assemelhando-se mais às outras formas de cobertura do solo (CAC e PAS). Esse fato pode ser devido ao arranjo e espaçamento das espécies utilizadas no sistema (Figura 1), que não permitiram a formação de um dossel fechado, resultando em menor cobertura do solo e, consequentemente, menor acúmulo de serrapilheira. Loss et al. (2009), Froufe et al. (2011) e Silva et al. (2011) também verificaram valores de atributos físicos do solo em SAFs semelhantes aos dos solos de sistemas agrícolas convencionais. Por outro lado, na pesquisa realizada por Carvalho et al. (2004), verifica-se que os sistemas agroflorestais podem 
apresentar valores de atributos físicos mais baixos, pois a Ds média na camada de 0 a $20 \mathrm{~cm}$ foi de $0,91 \mathrm{Mg} \mathrm{m}^{-3}$.

Os valores médios de macroporosidade (Tabela 3) indicam prováveis limitações à aeração do solo. $\mathrm{Na}$ camada de 0 a $20 \mathrm{~cm}$ estão muito próximos de $0,10 \mathrm{~m}^{3} \mathrm{~m}^{-3}$ e na camada de 20 a $40 \mathrm{~cm}$ estão abaixo deste valor, sendo considerado crítico para as plantas, de acordo com Pagliai et al. (2003).

Tabela 3. Macroporosidade (Map), microporosidade (Mip) e porosidade total $(\mathrm{Pt})$ em diferentes sistemas de uso do solo em duas profundidades em Ouro Preto do Oeste, RO.

\begin{tabular}{|c|c|c|c|c|c|c|}
\hline \multirow{2}{*}{$\begin{array}{c}\text { Atributos } \\
\left(\mathbf{m}^{3} \mathbf{m}^{-3}\right)\end{array}$} & \multirow{2}{*}{$\begin{array}{l}\text { Profundidades } \\
\text { (cm) }\end{array}$} & \multicolumn{4}{|c|}{ Sistemas de uso do solo } & \multirow{2}{*}{$\begin{array}{l}\text { CV } \\
(\%)\end{array}$} \\
\hline & & SAF & CAC & PAS & FLN & \\
\hline \multirow{2}{*}{ Map } & $0-20$ & $0,12^{\mathrm{ns}}$ & $0,12^{\mathrm{ns}}$ & $0,10^{\text {ns }}$ & $0,13^{\text {ns }}$ & 29,59 \\
\hline & $20-40$ & $0,05^{\text {ns }}$ & $0,04^{\mathrm{ns}}$ & $0,05^{\text {ns }}$ & $0,07^{\mathrm{ns}}$ & 28,22 \\
\hline \multirow{2}{*}{ Mip } & $0-20$ & $0,27 b$ & $0,32 \mathrm{a}$ & $0,32 \mathrm{a}$ & $0,34 \mathrm{a}$ & 6,69 \\
\hline & $20-40$ & $0,29 \mathrm{c}$ & $0,38 \mathrm{a}$ & $0,35 b$ & $0,37 \mathrm{a}$ & 5,62 \\
\hline \multirow{2}{*}{$\mathrm{Pt}$} & $0-20$ & $0,39 b$ & $0,44 b$ & $0,42 b$ & $0,47 \mathrm{a}$ & 7,70 \\
\hline & $20-40$ & $0,34 \mathrm{c}$ & $0,42 \mathrm{a}$ & $0,40 \mathrm{~b}$ & $0,44 a$ & 5,17 \\
\hline
\end{tabular}

Em que: $\mathrm{SAF}=$ sistema agroflorestal multiestratificado; $\mathrm{CAC}=$ cacau sob manejo agroecológico; PAS= pastagem; FLN= floresta natural, $\mathrm{CV}$ = coeficiente de variação. Médias na linha, seguidas de mesma letra, não diferem pelo teste de Sccott-Knott a 5\% de probabilidade; ${ }^{\text {ns }}=$ não significativo.

Acredita-se que o comportamento da macroporosidade esteja relacionado aos valores de Ds observados neste estudo. Richart et al. (2005) e Matias et al. (2009) reforçam essa ideia ao explicarem que, de modo geral, a Ds apresenta relação inversamente proporcional à macroporosidade. Esse comportamento pode ser confirmado pela correlação negativa entre Ds e macroporosidade (Tabela 4).

Nota-se que os menores valores de microporosidade, são observados no tratamento SAF nas duas camadas avaliadas (Tabela 3), pois conforme observado na Tabela 1, o SAF apresenta maior percentual de areia e nos tratamentos CAC, PAS e FLN, as médias para este atributo não diferem entre si, exceto na camada de 20 a $40 \mathrm{em}$ que pastagem difere de todos os tratamentos. Sabe-se que a microporosidade do solo é definida, principalmente, por processos de formação do solo e material de origem, entretanto a compactação do solo (aumento da Ds) pode reduzir a macroporosidade e elevar a micro (Viana et al., 2011).

Pesq. flor. bras., Colombo, v. 37, n. 89, p. 19-27, jan./mar. 2017
Tabela 4. Matriz de correlação dos atributos físicos e dos teores de carbono orgânico (COS) em duas profundidades do solo em Ouro Preto do Oeste, RO.

\begin{tabular}{|c|c|c|c|c|c|}
\hline $\begin{array}{l}\text { Profundidades } \\
\text { (cm) }\end{array}$ & Atributos & $\underset{\left(\mathbf{m}^{3} \mathbf{m}^{-3}\right)}{\operatorname{Mip}}$ & $\underset{\left(\mathbf{m}^{3} \mathbf{m}^{-3}\right)}{\text { Map }}$ & $\begin{array}{c}\mathbf{P t} \\
\left(\mathbf{m}^{3} \mathbf{m}^{-3}\right)\end{array}$ & $\begin{array}{c}\text { Teor de } \\
\text { COS } \\
\left(\mathrm{g} \mathrm{kg}^{-1}\right)\end{array}$ \\
\hline \multirow{8}{*}{$0-20$} & Ds & $-0,44^{\mathrm{ns}}$ & $-0,67 * *$ & $-0,86^{* *}$ & $-0,49 *$ \\
\hline & $\left(\mathrm{Mg} \mathrm{m}^{-3}\right)$ & $(>0,05)$ & $(<0,01)$ & $(<0,01)$ & $(<0,05)$ \\
\hline & Mip & & $-0,15^{\mathrm{ns}}$ & $0,63 * *$ & $0,66^{* *}$ \\
\hline & $\left(\mathrm{m}^{3} \mathrm{~m}^{-3}\right)$ & & $(0,51)$ & $(<0,01)$ & $(<0,01)$ \\
\hline & Map & & & $0,67 * *$ & $0,08^{\text {ns }}$ \\
\hline & $\left(\mathrm{m}^{3} \mathrm{~m}^{-3}\right)$ & & & $(<0,01)$ & $(0,72)$ \\
\hline & $\mathrm{Pt}$ & & & & $0,56^{* *}$ \\
\hline & $\left(\mathrm{m}^{3} \mathrm{~m}^{-3}\right)$ & & & & $(<0,01)$ \\
\hline \multirow{8}{*}{$20-40$} & Ds & $-0,54^{*}$ & $-0,52 *$ & $-0,71 * *$ & $-0,01^{\mathrm{ns}}$ \\
\hline & $\left(\mathrm{Mg} \mathrm{m}^{-3}\right)$ & $(<0,05)$ & $(<0,05)$ & $(<0,01)$ & $(0,96)$ \\
\hline & Mip & & $-0,02^{\mathrm{ns}}$ & $0,91 * *$ & $0,16^{\mathrm{ns}}$ \\
\hline & $\left(\mathrm{m}^{3} \mathrm{~m}^{-3}\right)$ & & $(0,93)$ & $(<0,01)$ & $(0,48)$ \\
\hline & Map & & & $0,40^{\mathrm{ns}}$ & $-0,26^{\mathrm{ns}}$ \\
\hline & $\left(\mathrm{m}^{3} \mathrm{~m}^{-3}\right)$ & & & $(0,08)$ & $(0,25)$ \\
\hline & $\mathrm{Pt}$ & & & & $0,04^{\mathrm{ns}}$ \\
\hline & $\left(\mathrm{m}^{3} \mathrm{~m}^{-3}\right)$ & & & & $(0,86)$ \\
\hline
\end{tabular}

Em que: Ds = Densidade do solo; Map = macroporosidade; Mip = microporosidade; $\mathrm{Pt}=$ Porosidade total; Teor de $\mathrm{COS}=$ teor de carbono orgânico do solo; ${ }^{\text {ns }}=$ não significativo; * $\mathrm{e}^{* *}=$ coeficiente de correlação de Pearson significativo ao nível de 1 e $5 \%$; números em itálico, abaixo do valor de cada correlação, representam sua probabilidade.

Para o atributo volume total de poros (Tabela 3), na camada de 0 a $20 \mathrm{~cm}$, todos os tratamentos diferiram da referência (FLN) que apresentou a maior média, e na camada de 20 a $40 \mathrm{~cm}$ apenas o tratamento CAC foi semelhante, destacando-se que no tratamento SAF verificou-se a menor média para volume total de poros em ambas profundidades. Possivelmente, o cultivo de espécies anuais anteriormente à implantação do SAF pode ter promovido alterações nos atributos físicos do solo e, diante disso, talvez seja necessário mais tempo para que as condições físicas do solo no SAF sejam revertidas com o atual arranjo e composição florística existente.

Assim como para a macroporosidade, as variações observadas para Pt podem estar associadas à densidade do solo. Souza Neto et al. (2008) contribuem no entendimento desse fenômeno ao afirmarem que a Ds é um atributo que pode indicar condições restritivas ao desenvolvimento radicular, em função da umidade do solo, da resistência à penetração e do espaço poroso do solo. 
Nesse contexto, diante da perspectiva ambiental, Silva et al. (2015) afirmam que os atributos físicos do solo (Ds, Mip, Map e Pt), são indicadores de serviços ambientais influenciados diretamente pelos sistemas de cobertura e, quando em estado crítico, podem reduzir o armazenamento de água e promover a degradação dos solos via escoamento superficial.

$\mathrm{Na}$ Tabela 5 encontram-se os teores de COS dos sistemas de uso e cobertura do solo estudados. Verificouse que na camada de 0 a $20 \mathrm{~cm}$ o sistema CAC apresentou a maior média, seguido de PAS, FLN e SAF. Esse comportamento se repete na camada 20 a $40 \mathrm{~cm}$, porém com o sistema FLN apresentando a segunda maior média para essa variável. De modo geral, os teores de COS no perfil do solo são consistentes com Corazza et al. (1999), que relatam que um terço da COS se encontra na camada superficial do solo, por conta da alta concentração de matéria orgânica nessa região, gerada pela deposição e decomposição do material vegetal.

Tabela 5. Teores de carbono orgânico do solo (COS) em diferentes sistemas de uso do solo e em duas profundidades em Ouro Preto do Oeste, RO.

\begin{tabular}{ccccccc}
\hline \multirow{2}{*}{$\begin{array}{c}\text { Atributo } \\
\left(\mathbf{g ~ k g}^{-1}\right)\end{array}$} & $\begin{array}{c}\text { Profundidades } \\
(\mathbf{c m})\end{array}$ & \multicolumn{4}{c}{ Sistemas de uso do solo } & CV \\
\cline { 3 - 5 } & & SAF & $\mathbf{C A C}$ & PAS & FLN & $(\%)$ \\
\hline \multirow{2}{*}{ Teor de COS } & $0-20$ & $7,60 \mathrm{~b}$ & $13,82 \mathrm{a}$ & $13,30 \mathrm{a}$ & $12,68 \mathrm{a}$ & 25,96 \\
& $20-40$ & $4,98 \mathrm{~b}$ & $9,80 \mathrm{a}$ & $7,36 \mathrm{a}$ & $8,51 \mathrm{a}$ & 25,04 \\
& $0-40$ & $12,58 \mathrm{~b}$ & $23,62 \mathrm{a}$ & $20,66 \mathrm{a}$ & $21,19 \mathrm{a}$ & 15,04 \\
\hline
\end{tabular}

Em que: $\mathrm{SAF}=$ sistema agroflorestal multiestratificado; $\mathrm{CAC}=$ cacau sob manejo agroecológico; PAS = pastagem; FLN = floresta natural. Médias na linha, seguidas de mesma letra, não diferem pelo teste de Sccott-Knott a 5\% de probabilidade.

Os teores de $\mathrm{C}$ encontrados para o SAF diferem dos demais tratamentos (Tabela 5), indicando menores estoque de COS em ambas profundidades. Esses resultados seguem a mesma tendência observada por Froufe et al. (2011) que relataram teores de COS na ordem de $18,55 \mathrm{~g} \mathrm{~kg}^{-1}$, em sistema agroflorestal multiestratificado com 16 anos de implantação. Os autores ainda mencionam que as práticas realizadas em SAFs são questionáveis, pois nem sempre promovem maiores estoques de COS. No entanto, deve-se levar em consideração que além do COS existe o acúmulo de carbono nos tecidos das plantas (raízes e parte aérea) e que práticas capazes de aumentar o aporte de matéria orgânica, por meio do uso de espécies com crescimento radicular agressivo ou ainda plantas de cobertura, são plausíveis para superar esse aspecto (Sá et al., 2015).
Resultados semelhantes aos teores de COS encontrados na pastagem e no cultivo de cacaueiros em relação ao SAF também foram observados por Sharrow \& Ismail (2004), que verificaram estoques de COS em pastagens e em plantios agrícolas jovens superiores aos encontrados em sistemas agroflorestais.

Em relação à pastagem, o comportamento para os estoques de COS é explicado por Costa Junior (2008) e Siqueira Neto et al. (2009), onde afirmam que os estoques de COS em pastagens podem atingir níveis muito próximos aos existentes em florestas naturais, pois o sistema radicular da gramínea, além de ser abundante e volumoso, é constantemente renovado, disponibilizando grande quantidade de matéria orgânica ao solo.

Os estoques de COS (Tabela 6) seguiram a mesma tendência observada para os teores de COS, exceto na profundidade de 20 a $40 \mathrm{~cm}$, na qual pode-se notar que a PAS se assemelhou ao SAF, ambos apresentando menor estocagem de COS. Nesse contexto, as considerações de Parron et al. (2015) auxiliam na explicação dos resultados obtidos, ao destacarem que os estoques de COS variam em função do tipo de solo, profundidade, clima, bioma e, principalmente, uso e manejo do solo.

Tabela 6. Estoques de carbono orgânico do solo (COS) em diferentes sistemas de uso do solo em diferentes profundidades em Ouro Preto do Oeste, RO.

\begin{tabular}{ccccccc}
\hline \multirow{2}{*}{$\begin{array}{c}\text { Atributo } \\
\left(\mathbf{M g ~ h a}^{-1}\right)\end{array}$} & $\begin{array}{c}\text { Profundidades } \\
(\mathbf{c m})\end{array}$ & \multicolumn{4}{c}{ Sistemas de uso do solo } & CV \\
\cline { 3 - 5 } & & SAF & CAC & PAS & FLN & (\%) \\
\hline & $0-20$ & $24,79 \mathrm{~b}$ & $42,71 \mathrm{a}$ & $41,30 \mathrm{a}$ & $36,07 \mathrm{a}$ & 24,15 \\
Estoque de & $20-40$ & $17,26 \mathrm{~b}$ & $31,06 \mathrm{a}$ & $22,31 \mathrm{~b}$ & $25,49 \mathrm{a}$ & 24,49 \\
COS & $0-40$ & $42,05 \mathrm{~b}$ & $73,76 \mathrm{a}$ & $63,61 \mathrm{a}$ & $61,56 \mathrm{a}$ & 14,24 \\
\hline
\end{tabular}

Em que: $\mathrm{SAF}=$ sistema agroflorestal multiestratificado $; \mathrm{CAC}=$ cacau sob manejo agroecológico; PAS = pastagem; FLN = floresta natural. Médias na linha, seguidas de mesma letra, não diferem pelo teste de Sccott-Knott a 5\% de probabilidade.

Ainda nessa perspectiva, Parron et al. (2015) afirmam que as determinações dos estoques de COS configuramse como valiosas ferramentas no âmbito das mudanças climáticas ao constituírem-se como importantes indicadores de serviços ambientais.

Apesar do menor estoque de COS verificado no SAF, cabe ressaltar que os sistemas agroflorestais, desde que adequadamente manejados, têm grande potencial para elevar os estoques de COS, pois apresentam diversidade vertical e horizontal. Portanto, existe a tendência dos estoques de COS serem semelhantes aos ecossistemas naturais e, em muitos casos, superiores àqueles 
encontrados em pastagens ou culturas de campo (Nair et al., 2009; Froufe et al., 2011; Rocha et al., 2014).

Além de contribuir para a redução do carbono atmosférico $\left(\mathrm{CO}_{2}\right)$, os SAFs podem ser protagonistas na recuperação de áreas degradadas em propriedades rurais (áreas de preservação permanente ou reserva legal), principalmente na agricultura familiar e, consequentemente, estimulam o incremento de áreas com boa capacidade produtiva. Essas ações podem diminuir a pressão sobre os fragmentos florestais remanescentes, pois o objetivo dos SAFs é produzir, harmonicamente, alimentos e produtos florestais, reduzindo-se os impactos à vegetação nativa (Rodrigues et al., 2007).

De modo geral, em relação aos atributos físicos do solo, acredita-se que se no SAF avaliado houvesse um maior adensamento entre as plantas que o compõem, ou ainda se fosse feito uso de plantas de cobertura que favorecessem o aporte de matéria orgânica na superfície, os valores de Map e Pt seriam próximos ou superiores aos encontrados nos demais ambientes avaliados, como pode ser observado por meio das correlações apresentadas entre esses atributos na Tabela 4.

\section{Conclusão}

Os diferentes usos e manejos influenciaram nos atributos físicos, teores e estoques de carbono do solo.

Os resultados referentes aos estoques de carbono indicaram comportamento esperado para o sistema agroflorestal, principalmente na camada superficial do solo $(0 \mathrm{~cm}$ a $20 \mathrm{~cm})$. No entanto, arranjos espaciais que favoreçam o aporte de matéria orgânica podem contribuir para a elevação dos estoques de carbono do solo em sistemas agroflorestais.

\section{Referências}

Altieri, M. Agroecologia: bases científicas para uma agricultura sustentável. 3. ed. Rio de Janeiro: Expressão Popular, 2012. 400 p.

Alvares, C. A. et al. Köppen's climate classification map for Brazil. Meteorologische Zeitschrift, v. 22, n. 6, p. 711-728, 2013. DOI: 10.1127/0941-2948/2013/0507.

Brasil. Ministério do Desenvolvimento Agrário. Plano territorial de desenvolvimento rural sustentado. Rio Branco: PESACRE, 2007. $135 \mathrm{p}$.

Carvalho, R. et al. Atributos físicos da qualidade de um solo sob sistema agroflorestal. Pesquisa Agropecuária Brasileira, v. 39, n. 11, p. 1153-1155, 2004. DOI: 10.1590/S0100-204X2004001100015.
Claessen, M. E. C. (Org.). Manual de métodos de análise de solos. 2. ed. Rio de Janeiro: EMBRAPA-CNPS, 1997.212 p. (EMBRAPACNPS. Documentos, 1).

Corazza, E. J. et al. Comportamento de diferentes sistemas de manejo como fonte ou depósito de carbono em relação à vegetação de cerrado. Revista Brasileira de Ciência do Solo, v. 23, n. 2, p. 425-432, 1999.

Costa Junior, C. Estoque de carbono e nitrogênio e agregação do solo sob diferentes sistemas de manejo agrícola. 2008. $140 \mathrm{f}$. Dissertação (Mestrado em Ciências) - Universidade de São Paulo, Piracicaba.

Donagema, G. K. et al. (Org.). Manual de métodos de análise de solos. 2. ed. Rio de Janeiro: Embrapa Solos, 2011. 230 p. (Embrapa Solos. Documentos, 132).

Froufe, L. C. M. et al. Potencial de sistemas agroflorestais multiestrata para sequestro de carbono em áreas de ocorrência de floresta Atlântica. Pesquisa Florestal Brasileira, v. 31, n. 66, p. 143-154, 2011. DOI: 10.4336/2011.pfb.31.66.143.

IBGE. Banco de dados georeferenciado dos recursos naturais da Amazônia legal. Rio de Janeiro, 2012. Disponível em: <ftp://geoftp. ibge.gov.br/cartas_e_mapas/>.Acesso em: 29 mar. 2015.

Loss, A. et al. Atributos químicos e físicos de um Argissolo VermelhoAmarelo em sistema integrado de produção agroecológica. Pesquisa Agropecuária Brasileira, v. 44, n. 1, p. 68-75, 2009. DOI: 10.1590/ S0100-204X2009000100010.

Magalhães, J. G. S. et al. Análise econômica de sistemas agroflorestais via uso de equações diferenciais. Revista Árvore, v. 38, n. 1, p. 7379, 2014. DOI: 10.1590/S0100-67622014000100007.

Matias, S. S. R. et al. Atributos físicos de um Latossolo Vermelho submetido a diferentes usos. Revista Ciência Agronômica, v. 40, n. 3, p. 331-338, 2009.

Nair, P. K. R. et al. Agroforestry as a strategy for carbono sequestration. Journal of Plant Nutrition and Soil Science, v. 172, n. 1, p. 10-23, 2009. DOI: 10.1002/jpln.200800030.

Pagliai, M. et al. Changes in some physical properties of a clay soil in Central Italy following the passage of rubber tracked and wheeled tractors of medium power. Soil and Tillage Research, v. 73, n. 1, 119-129, 2003. DOI: 10.1016/S0167-1987(03)00105-3.

Parron, L. M. et al. Estoques de carbono no solo como indicador de serviços ambientais. In: Parron, L. M. et al. Serviços ambientais em sistemas agrícola e florestais do Bioma Mata Atlântica. Brasília, DF: Embrapa, 2015. p. 71-83.

Pezarico, C. R. et al. Indicadores de qualidade do solo em sistemas agroflorestais. Revista de Ciências Agrárias, v. 56, n. 1, p. 40-47, 2012.

Richart, A. et al. Compactação do solo: causas e efeitos. Semina: Ciências Agrárias, v. 26, n. 3, p. 321-344, 2005.

Rocha, G. P. et al. Estoque de carbono em sistemas agroflorestais no Norte de Minas Gerais. Ciência Rural, v. 44, n. 7, p. 1197-1293, 2014.

Rodrigues, E. R. et al. Avaliação econômica de Sistemas Agroflorestais implantados para recuperação de reserva legal no Pontal do Paranapanema, São Paulo. Revista Árvore, v. 31, n. 5, p. 941-948, 2007. DOI: 10.1590/S0100-67622007000500018. 
Sá, J. C. M. et al. Carbon depletion by plowing and its restoration by no-till cropping systems in oxisols of subtropical and tropical agro-ecoregions in brazil. Land Degradation \& Development, v. 26, n. 6, p. 531-543, 2015. DOI: 10.1002/ldr.2218.

Sato, F. A. Balanço hídrico na cultura do cafeeiro (Coffea arábica L.). 2005. 100 f. Dissertação (Mestrado em Engenharia Agrícola) Universidade Federal de Lavras, UFLA, Lavras.

Santos, H. G. dos et al. Sistema brasileiro de classificação de solos. 3. ed. Brasília, DF: Embrapa, 2013. 353 p.

Sharrow, S. H. \& Ismail, S. Carbon and nitrogen storage in agroforests, tree plantations, and pastures in western Oregon, USA. Agroforestry Systems, v. 60, n. 2, p. 123-160, 2004. DOI: 10.1023/B:AGFO.0000013267.87896.41.

Silva, A. H. et al. Atributos físicos do solo e escoamento superficial como indicadores de serviçoes ambientais. In: Parron, L. M. et al. Serviços ambientais em sistemas agrícola e florestais do Bioma Mata Atlântica. Brasília, DF: Embrapa, 2015. p. 71-83.

Silva, D. C. et al. Atributos do solo em sistemas agroflorestais, cultivo convencional e floresta nativa. Revista de Estudos Ambientais, v. 13 , n. 1, p. 77-86, 2011.
Silva, L. M. et al. Comparação de métodos para a determinação de carbono em solos no estado do Acre. In: REUNIÃO DE CIÊNCIA DO SOLO DA AMAZÔNIA OCIDENTAL, 2. 2014. Porto Velho. Anais... Porto Velho: Sociedade Brasileira de Ciências do Solo, 2014. p. 39-42.

Silva, R. F. et al. Atributos físicos e teor de matéria orgânica na camada superficial de um Argissolo vermelho cultivado com mandioca sob diferentes manejos. Revista Brasileira de Ciência do Solo, v. 32, n. 6, p. 2435-2441, 2008.

Siqueira Neto, M. et al. Carbono total e atributos químicos com diferentes usos do solo no Cerrado. Acta Scientiarum. Agronomy, v. 31, n. 4, p. 709-717, 2009. DOI: 10.4025/actasciagron.v31i4.792.

Souza Neto, E. L. et al. Atributos físicos do solo e produtividade de milho em resposta a culturas de pré safra. Pesquisa Agropecuária Brasileira, v. 43, n. 2, p. 255-260, 2008. DOI: 10.1590/S0100204X2008000200015.

Veldkamp, E. Organic carbon turnover in three tropical soils under pasture after deforestation. Soil Science Society of America Journal, v. 58 , p. $175-180,1994$. DOI: $10.2136 /$ sssaj1994.03615995005800 010025x.

Viana, E. T. et al. Atributos físicos e carbono orgânico em latossolo vermelho sob diferentes sistemas de uso e manejo. Revista Brasileira de Ciência do Solo, v. 35, n. 1, p. 2105-2114, 2011. 
